\title{
A Study of Crystallographic and DC Electrical Characteristics of PPy/Ag Nanocomposites
}

\author{
Mohammed Abdilridha Salman, Salma Mohammad Hassan \\ Department of Physics, College of Science, University of Baghdad, Baghdad, Iraq \\ E-mail: salma.mhammed@yahoo.com
}

Corresponding author: mohammed.a.salman@outlook.com

\begin{abstract}
Polypyrrole and polypyrrole/silver nanocomposites were fabricated by in-situ polymerization employing Ammonium Persulphate as an oxidizing agent. Nanocomposites were synthesized by combining polypyrrole and silver nanoparticles in various weight percentages $(0.1 \%, 0.5 \%, 3 \%, 5 \%$ and $7 \%$ wt.). Crystallographic data were collected using X-ray diffraction. PPy particles were found to have an orthorhombic symmetry. In contrast, PPy/Ag nanocomposites were reported to have monoclinic structure. The crystallite size was determined by XRD using Scherrer equation and considered to be within $49 \mathrm{~nm}$ range. DC conductivity of pelletized samples was evaluated in the temperature range of $323 \mathrm{k}$ to $453 \mathrm{k}$. The conductivity displayed an increase when the temperature is increased. Activation energies were determined from plots of Arrhenius for all nanocomposites. The findings indicated that the activation energy decrease with increasing the weight percentage of Ag nanoparticles in the nanocomposites.
\end{abstract}

Key words

Polypyrrole, silver nanoparticles, nanocomposites, crystallographic, $D C$ electrical conductivity.

\section{Article info.}

Received: Oct. 2020

Accepted: Dec. 2020

Published: Jun. 2021

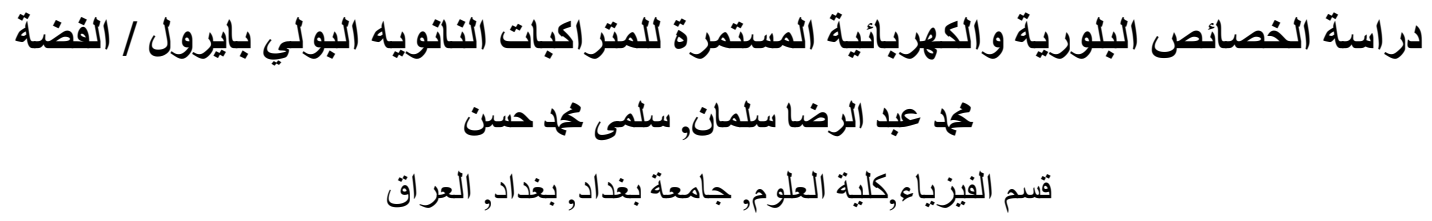




\section{Introduction}

Due to its unique characteristics and wide range of applications in various electronic devices, conductive paint, drug delivery, rechargeable batteries and sensors, etc., nanocomposites have been one of the most thoroughly investigated materials worldwide in the last two decades. Conducting polymers have effective electrical conductivity, but they are sensitive to chemical exposure and have poor mechanical properties. Metal nanoparticles, on the other hand, have good mechanical properties $[1,2]$. Due to its high electrical conductivity, enhanced stability, structural properties and elevated flexibility in its preparation, polypyrrole is the most widely tested conducting polymer across all the known conducting polymers [3]. Metal nanoparticles, in particular gold, silver, platinum and copper, have been the subject of great attention due to its unique electronic, optical, catalytic and thermal properties, as well as their significant applications in multiple fields [4]. Conductive polymers nanocomposites equipped with metals nanoparticles offers exciting methods for exploring the probability of constructing many devices and incorporating their functionality into many disciplines [5]. Therefore, metallic nanoparticles and polymers have evoked a variety of researchers' involvement in synthesizing nanocomposite materials [6]. In fact, PPy-Ag nanocomposites demonstrate superior efficiency in optoelectronics and catalytic sensing in comparison to pure PPy nanocomposites [7]. The production of PPy/Ag nanocomposites is therefore an innovative task for researchers. Numerous types of synthesis have been researched for the development of these nanocomposites. For instance, Bedre et al. used interfacial polymerization to fabricate conducting polypyrrole silver (PPy-AgNC) nanocomposites [8]. Also, Skodova et al. Polypyrrole nanotubes have been synthesized by oxidation of pyrrole with iron (III) chloride in the existence of methyl orange. Successively, they were used to reduce silver ions to silver nanoparticles [9]. Not to mention, Gupta et al. reported a simple electrochemical method of synthesis of polypyrrole/silver (PPy/Ag) nanocomposite [10].

This study was conducted to Synthesise PPy/Ag nanocomposites via in-situ polymerization. Polypyrrole and silver nanocomposites were synthesised using the same method for different compositions. The prepared PPy/Ag nanocomposites were analyzed by XRD, and the electrical conductivity of PPy/Ag nanocomposite was investigated using two probe method.

\section{Experimental work \\ Reagents and materials}

Various materials have been used to synthesis both PPy and PPy/Ag nanocomposites. These materials include: (i) pyrrole as a monomer, supplied by FlukaAG doubly distilled and cooled in icy water before use (ii) Ammonium peroxydisulfate (APS) functioned as an oxidant, provided by (Central Drug House, India) and used without any modifications (iii) Silver nanoparticles (99.9\% 10nm) were obtained from American Elements and were used as delivered (iv) Hydrochloric acid to be diluted and used as cleaning solution for the sample.

\section{Synthesis of polypyrrole}

After being weighed by a 4-digit balance, an amount of $1.34 \mathrm{ml}$ of pyrrole was placed in a beaker and diluted with $50 \mathrm{ml}$ of distilled water. By doing so, a one mole concentration was obtained. After that, this mixture was poured in a 3 neck round bottom flask, kept under a constant stirring and remained under ice bath at $3{ }^{\circ} \mathrm{C}$ for 15 minutes. Meanwhile, another solution of $1 \mathrm{M}$ of ammonium peroxydesulphate (APS) 
was prepared by adding $50 \mathrm{ml}$ of distilled water to $4.58 \mathrm{~g}$ of such material. Similarly, this solution was retained under constant stirring and at low temperature. The APS solution was then added drop wise to the pyrrole solution. It was observed that the color of the solution had been changed to dark black color after being transparent with the appearance of granules. A digital thermometer was dipped into the solution to monitor the temperature change. Soon, the temperature rose from 3 to $17{ }^{\circ} \mathrm{C}$ in less than 10 seconds and started to drop gradually. The polymerization process was left to undertake for a period of $5 \mathrm{~h}$ under constant stirring at $3{ }^{\circ} \mathrm{C}$. In addition, the solution was left to settle for 20 hours.

In the next day, this mixture was filtered under vacuum. Meanwhile, $0.6 \mathrm{ml}$ of hydrochloric acid was added to $50 \mathrm{ml}$ of water and used to wash the mixture. Later, a volume of $50 \mathrm{ml}$ of distilled water was added to the above filtered compound for washing purposes. The resulting compound from the filtration process was dried by leaving it in the oven at $80{ }^{\circ} \mathrm{C}$ for 4 hours. After making sure that the powder was humidity free, it was crushed by mortar into fine powder for further diagnostic analyses. Fig. 1 demonstrate the black color of PPy powder.

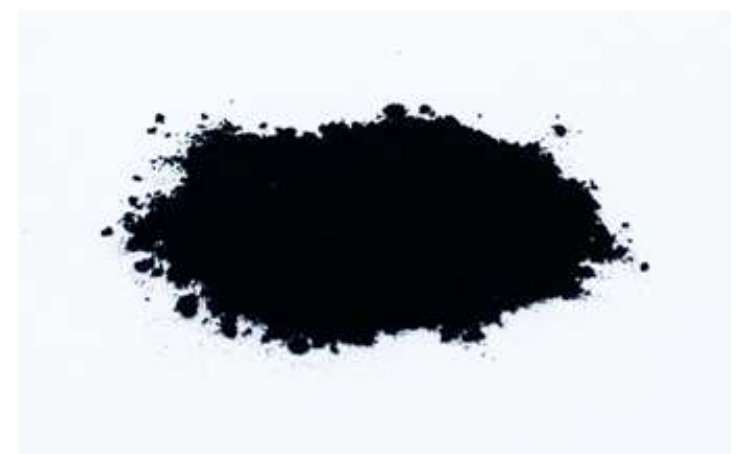

Fig. 1: Image of PPy nanoparticles powder.

\section{Synthesis of PPy/Ag nanocomposites}

In a similar manner, the distilled pyrrole was used in the preparation process of $\mathrm{PPy} / \mathrm{Ag}$ nanocomposites as follows.

$\mathrm{Ag}$ nanoparticles of weight percentages $(0.1 \%, 0.5 \%, 3 \%, 5 \%$ and $7 \%$ wt.) were weighed by a 4-digit balance in a glove box filled with nitrogen to ensure oxygen-free environment. $1.34 \mathrm{ml}$ of pyrrole has been weighed and kept in a closed beaker. A 50 $\mathrm{ml}$ of distilled water was added to pyrrole to acquire one mole concentration. Thereafter, the solution was poured in a 3 neck round bottom flask that was placed under ice bath at $3{ }^{\circ} \mathrm{C}$ with constant stirring for 15 minutes. Afterwards, silver nanoparticles were added to the diluted pyrrole and kept under stirring for 15 minutes. Next, another solution of $1 \mathrm{M}$ of ammonium peroxydesulphate (APS) was prepared by dissolving $4.58 \mathrm{~g}$ into a $50 \mathrm{ml}$ of water. This solution was kept under $3{ }^{\circ} \mathrm{C}$ temperature. Soon after, the APS solution was added drop wise to the pyrrole solution. This process is described in Fig.2. 


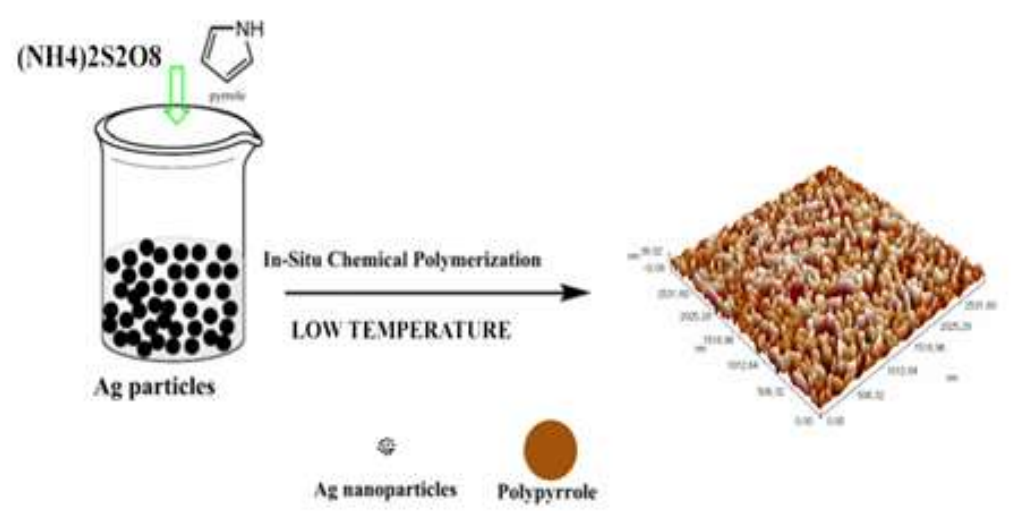

Fig. 2: Schematic representation of formation mechanism of PPy/Ag nanocomposites. Electrical characterization

The fine PPy and PPy/Ag nanocomposites powders were compressed into a pellet with $13.5 \mathrm{~mm}$ diameter and $3 \mathrm{~mm}$ thickness at a pressure of $8 \mathrm{ton} / \mathrm{cm}^{2}$. The pelletized samples were then brushed with a conductive silver paste and dried under vacuum until electrical measurements were taken. The resistance was evaluated by two probes with a range of temperatures of $323.15 \mathrm{k}$ to $453.15 \mathrm{k}$. Electrical conductivity was measured by the following formula:

$$
\sigma=\frac{L}{R * A}
$$

where $\sigma$ is the conductivity in $\mathrm{S} / \mathrm{cm}, \mathrm{R}$ is the resistance $(\mathrm{Ohm}), \mathrm{L}$ is the distance between electrodes $(\mathrm{cm})$, and $\mathrm{A}$ is the area in $\mathrm{cm}^{2}$.

\section{Results and discussion \\ Crystallographic analysis}

PPy and PPy /Ag nanocomposites XRD patterns verified the synthesis of PPy nanoparticles and the integration of Ag nanoparticles in PPy matrix as presented in Fig.3. The broad peak at $2 \theta=23.588^{\circ}$ is due to the amorphous nature of PPy. The four sharp Ag peaks at $2 \theta=32.44^{\circ}, 38.31^{\circ}, 46.47^{\circ}$ and $54.96^{\circ}$ that can be assigned to (6 02 2), (3 14 4), ( 31 - 1 -6) and (4 20 ), respectively which was confirmed by JCPDS file no. 96-150-8431. As can be noticed from the XRD patterns, the PPy nanoparticles have an orthorhombic symmetry. In contrast, PPy/Ag nanocomposites were reported to have monoclinic structure. In addition, the average particle size, estimated using the most intense diffraction peaks and Scherrer equation:

$$
D=\frac{K \lambda}{\beta \cos \theta}
$$

where $\mathrm{D}$ is the grain size $(\mathrm{nm}), \mathrm{K}=0.9$ (Scherrer constant), $\lambda=0.15406 \mathrm{~nm}$ (wavelength of the x-ray sources), $\boldsymbol{\beta}$ is the FWHM (in radians), and $\boldsymbol{\theta}$ is the Peak position (in radians). The crystallites size varied between $36 \mathrm{~nm}$ and $82 \mathrm{~nm}$. These results have a number of similarities with M. F. Ghadim et al. [11] findings and also confirms previous findings $[9,12,13]$. 


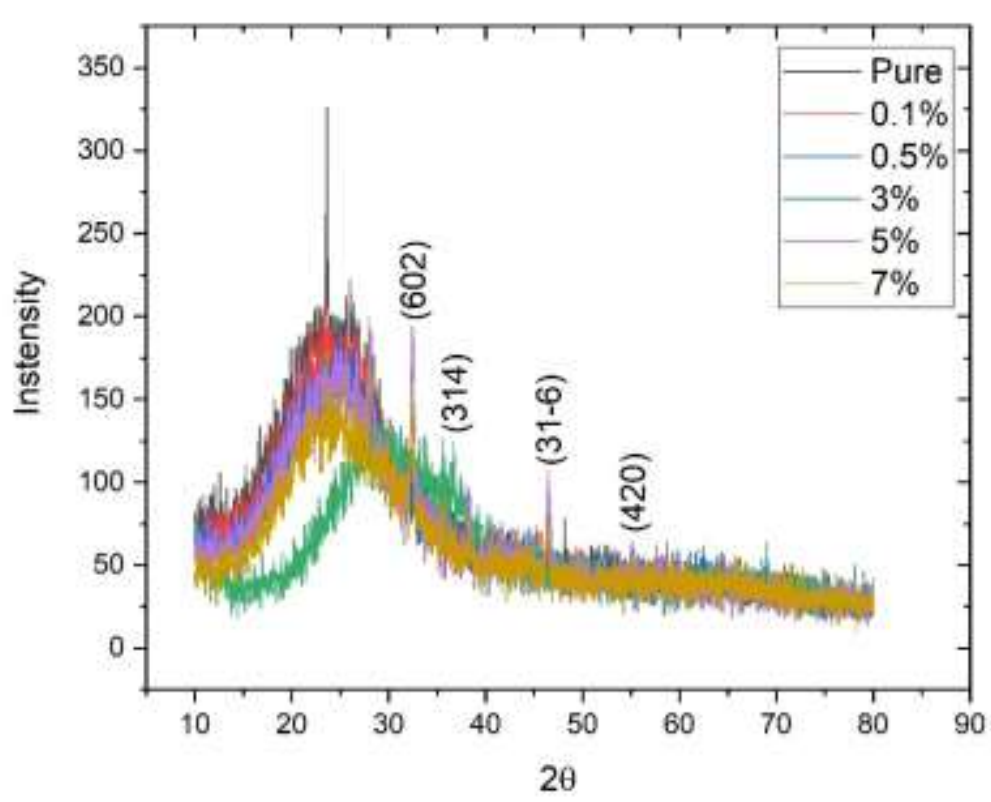

Fig. 3: X-ray diffraction pattern of PPy and PPy/Ag nanocomposites $(0.1 \%, 0.5 \%, 3 \%, 5 \%$ and $7 \% w t$.$) .$

\section{DC conductivity}

In order to establish the current conductivity mechanism in pure PPy and PPy/Ag nanocomposites, the variance of DC electrical conductivity in the temperature range from $323.15 \mathrm{k}$ to $453.15 \mathrm{k}$ was investigated. The conductivity of the formulated PPy/Ag $(0.1 \%, 0.5 \%, 3 \%, 5 \%$ and $7 \%$ wt.) nanocomposites was observed to be $7.813 \times 10^{-5}, 8.621 \times 10^{-5}, 1.00 \times 10^{-4}, 1.250 \times 10^{-4}$ and $1.471 \times 10^{-4} \mathrm{~S} / \mathrm{cm}$ respectively, while that of pure PPy was found to be $7.143 \times 10^{-5} \mathrm{~S} / \mathrm{cm}$ at $323 \mathrm{k}$ temperature. The improvement in electrical conductivity arises based on electronic tunneling via the $\mathrm{Ag}$ nanoparticles [14].

\section{Activation energy}

The variation of electrical conductivity against 1000/T of PPy and PPy/Ag nanocomposites at various temperatures can be presented by Arrhenius equation which can be illustrated by the following equation [14]:

$$
\sigma=\sigma_{0} \exp \left[-\frac{E_{a}}{k_{B} T}\right]
$$

Where $(\sigma)$ is the electrical conductivity, $\left(\sigma_{0}\right)$ is the conductivity in infinite temperature which is constant, $\left(\mathrm{E}_{\mathrm{a}}\right)$ is the activation energy, $\mathrm{k}_{\beta}$ is Boltzmann constant, $\mathrm{T}$ is the temperature (K). Ln $\sigma$ and $1000 / \mathrm{T}$ plots are considered to be linear. This suggests that the regular band model can be used for semiconduction in PPy and PPy/Ag nanocomposites [15]. Activation energy is approximated mostly from slope of the Arrhenius plot of $\ln \sigma$ versus (1000/T) [15]:

$$
\left(\mathbf{E}_{\mathbf{a}}\right)=- \text { slope } \times \mathbf{k}
$$

The slope is negative and the activation energy has a positive value. This can be calculated from Fig.4 for different compositions. Results infers that the activation 
energy for PPy is $0.033 \mathrm{eV}$ and it decreases until reaching $0.025 \mathrm{eV}$ for PPy/Ag (7\%wt.). This verifies the electronic tunneling because of the Ag NPs [14].

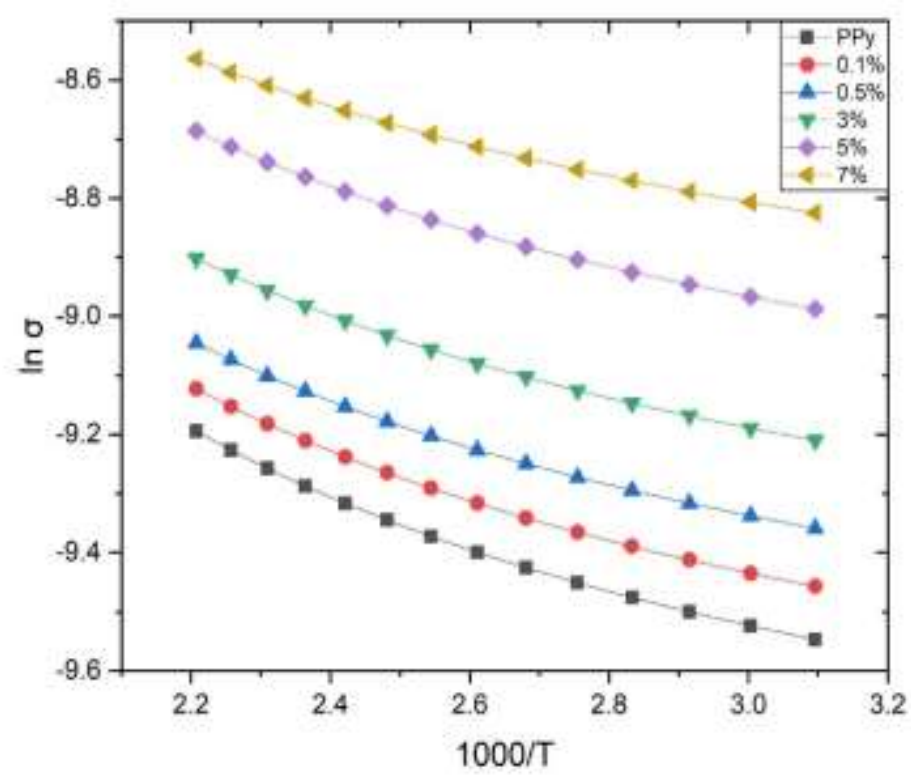

Fig.4: Variation of DC conductivity with temperature of PPy and PPy-Ag nanocomposite.

The relationship between activation energy and the added Ag nanoparticles in PPy matrix can be illustrated in Fig.5. It is important to note how the DC conductivity is dependent on temperature as exhibited by Arrhenius equation, and how the $\mathrm{Ag}$ nanoparticles plays an effective role in increasing the electrical conductivity.

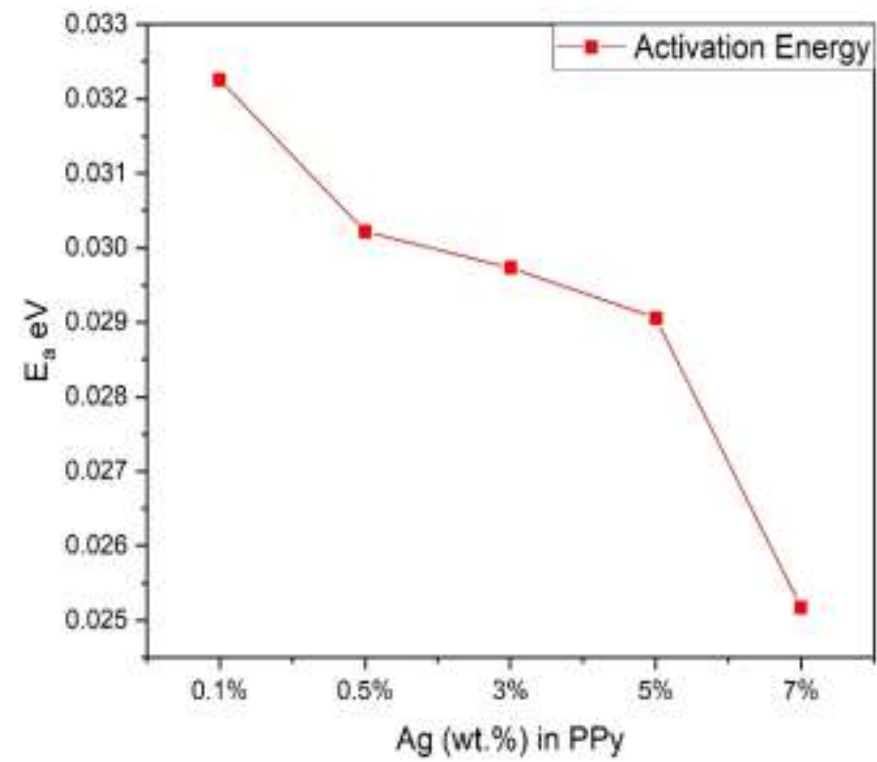

Fig.5: Variation of activation energy with Ag weight percentage. 


\section{Conclusions}

This work presented an explanatory strategy, the PPy and PPy/Ag nanocomposites were successfully synthesized via in situ chemical oxidative polymerization of pyrrole, in the presence of Ag NPs. The results of XRD analysis indicated that the PPy/Ag $(0.1 \%, 0.5 \%, 3 \%, 5 \%$ and $7 \%$ wt.) nanocomposites were successfully synthesized. Ag NPs with an average size of $\sim 10 \mathrm{~nm}$ were embedded in the PPy matrix. The average grain size varied between $36 \mathrm{~nm}$ and $82 \mathrm{~nm}$. In addition, the electrical conductivity of nanocomposites of PPy and PPy / Ag was tested using the two-probe approach and was found to be greater than that of pure PPy. It was also observed that the activation energy (Ea) decreased from $0.033 \mathrm{eV}$ to $0.025 \mathrm{eV}$ for PPy/Ag (0.07\% wt.) nanocomposites, in contrast with that of pure PPy. The fabricated nanocomposites can be used to make gas sensors working at room temperature as a possible material or, it can be used as a conductive paint to in some circumstances where the metallic materials cannot be used. Also, by increasing the silver content, PPy/Ag based nanocomposites can be used as electrodes for supercapacitors

\section{Acknowledgement}

The authors wish to thank Assist. Prof. Amer Faisal Abdilamer from department of Physics, college of Science, University of Baghdad for his valuable assistance and helpful advice throughout this work.

\section{References}

[1] K. Suri, S. Annapoorni, R. P. Tandon, Bull. Mater. Sci., 24, 6 (2001) 563-567.

[2] H. Arami, M. Mazloumi, R. Khalifehzadeh, S. H. Emami, S. K. Sadrnezhaad, Mater. Lett., 61, 22 (2007) 4412-4415.

[3] V.V. Tat'yana and O.N. Efimov, Russ. Chem. Rev., 66, 5 (1997) 443-454.

[4] S.H. Lee, J.H. Sung, T.H. Park, Ann Biomed Eng, 40 (2012) 1384-1397.

[5] P. H. C. Camargo, K. G. Satyanarayana, F. Wypych, Mater. Res., 12, 1 (2009) 139.

[6] H. Takeoka, H. Hamasaki, Y. Harada, Y. Nakamura, S. Fujii, Colloid Polym. Sci., 293, 5 (2015) 1483-1493.

[7] K. H. Kate, S. R. Damkale, P. K. Khanna, G. H. Jain, J. Nanosci. Nanotechnol., 11, 9 (2011) 7863-7869.

[8] M. D. Bedre, S. Basavaraja, R. Deshpande, D. S. Balaji, A. Venkataraman, Int. J. Polym. Mater., 59, 8 (2010) 531-543.

[9] J. Škodová, D. Kopecký, M. Vrňata, M. Varga, J. Prokeš, M. Cieslar, P. Bober, J. Stejskal, Polym. Chem., 4, 12 (2013) 3610-3616.

[10] R. Gupta, K. Jayachandran, J. S. Gamare, B. Rajeshwari, S. K. Gupta, J. V Kamat, J. Nanoparticles, 2015 (2015) 1-8.

[11] M. F. Ghadim, A. Imani, G. Farzi, J. Nanostructure Chem., 4, 2 (2014) 101-106.

[12] S. S. Shinde, A. K. Jayant, V. K. Milind, Int. J. Innov. Res. Sci. Eng. Technol., 3, 6 (2014) 14021-14026.

[13] E. Pinter, R. Patakfalvi, T. Fülei, Z. Gingl, I. Dekany, C. Visy, J. Phys. Chem. B, 109, 37 (2005) 17474-17478.

[14] K. Gupta, P. C. Jana, A. K. Meikap, Synth. Met., 160, $13-14$ (2010) 1566-1573.

[15] V. S. Shanthala, S. N. Shobha Devi, M. V Murugendrappa, J. Asian Ceram. Soc., 5,3 (2017) 227-234. 